\title{
Limite Clássico da Mecânica Quântica
}

\author{
Classical Limit of Quantum Mechanics \\ A. O. Bolivar* \\ Instituto Cultural Eudoro de Sousa, \\ Grupo “Mário Schönberg” de Estudos em Física-Matemática-Filosofia, \\ Ceilândia, 72221-970, Cx. P. 7316, D.F, Brazil.
}

Recebido em 16 de agosto, 2002. Aceito em 17 de abril, 2003.

\begin{abstract}
Neste trabalho, apresentamos um procedimento alternativo para o cálculo do limite clássico $(\hbar \rightarrow 0)$ das equações de movimento da mecânica quântica. Comparamos nosso método com o teorema de Ehrenfest, a aproximação WKB e com o potencial quântico de Bohm $Q \rightarrow 0$. Segue, também, a tradução de um artigo do Einstein sobre a problemática da conexão entre as teorias clássica e quântica.
\end{abstract}

In this work we propose an alternative procedure to calculate the classical limit $(\hbar \rightarrow 0)$ of the quantum mechanical equations of motion. We compare our method with the Ehrenfest theorem, the WKB approximation and the Bohm quantum potential $Q \rightarrow 0$. We also translate an Einstein's paper about the relation between quantum and classical theories.

\section{Introdução}

Em vários livros-texto [1], a conexão entre a mecânica quântica e a clássica é realizada ao se fazer uso do teorema de Ehrenfest [2] e/ou da approximação WKB [3-5]. O primeiro consiste em deduzir as equações

$$
\begin{gathered}
\frac{d\langle p\rangle}{d t}=-\frac{\partial V(\langle q\rangle, t)}{\partial q} \\
\frac{d\langle q\rangle}{d t}=\frac{\langle p\rangle}{m}
\end{gathered}
$$

a partir da equação de Schrödinger

$$
-\frac{\hbar^{2}}{2 m} \frac{\partial^{2} \psi}{\partial q^{2}}+V(q, t) \psi=\imath \hbar \frac{\partial \psi}{\partial t}
$$

por meio da relação

$$
\langle A\rangle=\int \psi^{\dagger} \hat{A} \psi d q
$$

para o valor esperado de uma dada observável física $A=$ $A(p, q, t)$ representada pelo operador $\hat{A}$. As equações (1) e (2) são válidas desde que o potencial externo $V$ varie vagarosamente dentro de um pacote de onda bem localizado $\psi_{P E}$ (Pacote de Ehrenfest). Assim, de acordo com os livros-texto [1], as equações de Newton são obtidas como limite clássico da equação de Schrödinger. No entanto, Ballentine et al. $[6,7]$ (veja também [8,9]) recentemente demonstraram que as condições de validade do teorema de Ehrenfest não são nem necessárias nem suficientes para caracterizar o limite clássico da mecânica quântica.
O método WKB [10-14], por sua vez, parte de uma dada solução

$$
\psi=e^{\imath\left[S+(\hbar / \imath) S_{1}+(\hbar / \imath)^{2} S_{2}+\ldots\right] / \hbar}
$$

da Eq.(3). Ao introduzirmos (4) em (3), ao assumirmos que o potencial externo $V$ seja suficientemente "suave" e varie muito devagar dentro do pacote WBK $\psi_{W K B}=e^{S_{1}} e^{\imath S / \hbar}$ ( $S_{1}$ and $S$ não têm $\hbar$ ), obtemos a equação de HamiltonJacobi

$$
\frac{\partial S}{\partial t}+\frac{1}{2 m}\left\{\frac{\partial S}{\partial q}\right\}^{2}+V(q, t)=0
$$

para a ação clássica $S=S(q, t)$. Embora a aproximação WKB forneça corretamente a Eq.(5) como limite clássico da equação de Schrödinger (3), tal processo de limite é incompatível com o princípio de superposição da mecânica quântica, isto é, a superposição de duas funções WKB $\psi^{\prime}=$ $e^{S_{1}}\left(e^{\imath S / \hbar}+e^{-\imath S / \hbar}\right)$ não pode ser representada pela função (4). Deste modo, não há nenhum limite clássico para esta classe de funções de onda. Além disso, há sistemas físicos que não possuem $\psi_{W K B}$ como solução aproximada. Isto parece ser uma forte restrição do método WKB.

\section{O potencial quântico de Bohm [15- 21]}

A representação de Madelung da mecânica quântica [15] é caracterizada pelas equações

$$
\frac{\partial \mathcal{S}}{\partial t}+\frac{1}{2 m}\left\{\frac{\partial \mathcal{S}}{\partial q}\right\}^{2}+Q(q, t)+V(q, t)=0,
$$

\footnotetext{
*Endereço atual: II. Institut für Theoretische Physik, Universität Stuttgart, Pfaffenwaldring 57/III, D-70550, Stuttgart, Germany.
} 


$$
\frac{\partial R^{2}}{\partial t}+\frac{1}{m} \frac{\partial}{\partial q}\left\{R^{2} \frac{\partial S}{\partial q}\right\}=0
$$

em que

$$
Q(q, t)=-\frac{\hbar^{2}}{2 m} \frac{1}{R} \frac{\partial^{2} R}{\partial q^{2}}
$$

é o potencial quântico de Bohm. As equações (6) e (7) são obtidas ao substituirmos

$$
\psi(q, t)=R(q, t) e^{\imath \mathcal{S}(q, t) / \hbar}
$$

na Eq.(3). $R=R(q, t)$ e $\mathcal{S}=\mathcal{S}(q, t)$ são funções dependentes de $\hbar$, em geral. Aqui o limite clássico da mecânica quântica é realizado ao usarmos o seguinte critério [17-21]

$$
Q \rightarrow 0
$$

Conseqüentemente, a equação de Hamilton-Jacobi quântica (6) se reduz à equação de Hamilton-Jacobi clássica.

Para ilustrar o uso da condição (9), consideremos três casos: uma partícula livre, um oscilador harmônico e uma partícula em uma caixa.

\section{Partícula livre $V=0$ :}

As soluções da equação de Schrödinger (3) são dadas por

$$
\psi_{1}=A e^{\imath\left(p q-p^{2} t / 2 m\right) / \hbar} \quad \text { ou } \quad \psi_{2}=A e^{-\imath\left(p q+p^{2} t / 2 m\right) / \hbar}
$$

em que $A$ e $p$ são constantes reais. É fácil verificar que $\psi_{1}$ ou $\psi_{2}$ leva a $Q=0$ e ao momentum $p=\partial \mathcal{S} / \partial q$, enquanto que a superposição $\psi=\left(\psi_{1}+\psi_{2}\right) / \sqrt{2}$ gera $Q=p^{2} / 2 m$ e $p=0$. Assim, de acordo com a teoria de Bohm uma partícula livre quântica é descrita pela mecânica clássica somente para os estados $\psi$ que não são funções superpostas.

\section{Oscilador harmônico $V=m \omega^{2} q^{2} / 2$ :}

As soluções estacionárias

$$
\psi_{n}(q)=c_{n} H_{n}(\xi q) e^{-\xi^{2} q^{2} / 2}
$$

$\operatorname{com} \xi=(m \omega / \hbar)^{1 / 2}, c_{n}=\xi /\left(\pi^{1 / 2} 2^{n} n !\right)^{1 / 2}$ e $H_{n}$ os polinômios de Hermite, geram o potencial quântico

$$
Q=\hbar \omega\left(n+\frac{1}{2}\right)-\frac{1}{2} m \omega^{2} q^{2}
$$

que é sempre diferente de zero. Portanto, o oscilador harmônico quântico não possui limite clássico. Ademais, a teoria de Bohm prevê que o sistema se encontra em repouso $(p=0)$ com energia total igual a $\hbar \omega(n+1 / 2)$.

\section{Partícula em uma caixa}

A equação de Schrödinger (3), satisfazendo as condições de contorno $\psi(q=-a, t)=\psi(q=a, t)=0$, fornece as soluções

$$
\psi_{n^{\prime}}(q, t)=(2 / a)^{1 / 2} \cos \left(\frac{n^{\prime} \pi}{a} q\right) e^{t \imath E_{n^{\prime}} / \hbar}
$$

com

$$
E_{n^{\prime}}=\frac{1}{2 m}\left(\frac{\hbar n^{\prime} \pi}{a}\right)^{2} ; \quad n^{\prime}=n+\frac{1}{2} \quad(n=0,1,2, \ldots) .
$$

O potencial quântico, associado com (13), é

$$
Q_{n^{\prime}}=\frac{1}{2 m}\left(\frac{\hbar n^{\prime} \pi}{a}\right)^{2}
$$

que se anula quando $\hbar \rightarrow 0$ (com $n^{\prime}$ fixo). Aqui, segundo a interpretação de Bohm a partícula está sempre em repouso $(p=0)$ no limite clássico. Quando $n^{\prime} \rightarrow \infty$ e $\hbar \rightarrow 0$, não há nenhum limite clássico $(Q \neq 0)$.

Devido às características incomuns inerentes ao método do Bohm, acima acentuadas, Cohn [22] e Rosen [23] propuseram esquemas alternativos para calcular o limite clássico da mecânica quântica a partir da representação de Madelung. Especificamente, Cohn sustenta o ponto de vista de que, embora o potencial quântico possa ser nulo, a identificação $p=\partial \mathcal{S} / \partial q$ não pode ser feita, em geral. Einstein [24] (ver Seção IV) e Ballentine [7] também enfatizam a não total adequabilidade do critério (9) para caracterizar o limite clássico da Eq.(6). Abaixo, mostramos que tal critério, de fato, não é necessário para obter a equação de HamiltonJacobi clássica.

\section{Nosso método}

Acima, enfatizamos que o teorema de Ehrenfest, a aproximação WKB e o método do potencial quântico de Bohm não são processos de limite clássico adequados, devido ao fato de não serem baseados explicitamente no limite $\hbar \rightarrow 0$.

Agora, queremos apresentar um método compatível com o princípio de superposição e enfatizar o limite $\hbar \rightarrow 0$ como o critério universal para calcular o limite clássico da mecânica quântica sem a necessidade de tomar o limite $\hbar \rightarrow$ 0 de soluções individuais $\psi$ da equação de Schrödinger.

Efetuamos a transformação unitária

$$
\psi^{\prime}=e^{-\imath G / \hbar} \psi
$$

sobre a equação de Schrödinger (3) $(G=G(q, t)$ é uma função sem $\hbar$ ) e obtemos

$$
\left\{\frac{-\hbar^{2}}{2 m} \frac{\partial^{2}}{\partial q^{2}}-\frac{\imath \hbar}{2 m}\left[\frac{\partial^{2} G}{\partial q^{2}}+2 \frac{\partial G}{\partial q} \frac{\partial}{\partial q}+2 m \frac{\partial}{\partial t}\right]+\left[\frac{1}{2 m}\left(\frac{\partial G}{\partial q}\right)^{2}+V+\frac{\partial G}{\partial t}\right]\right\} \psi^{\prime}=0
$$


Notamos que

$$
\begin{gathered}
\lim _{\hbar \rightarrow 0}\left\{\hat{p}^{\prime}=e^{-\imath G / \hbar}\left(-\imath \hbar \frac{\partial}{\partial q}\right) e^{\imath G / \hbar}\right\}=\frac{\partial G}{\partial q} \equiv p \\
\lim _{\hbar \rightarrow 0}\left\{{\hat{p^{\prime}}}^{2}=e^{-\imath G / \hbar}\left(-\imath \hbar \frac{\partial}{\partial q}\right)^{2} e^{\imath G / \hbar}\right\}=\left(\frac{\partial G}{\partial q}\right)^{2} \equiv p^{2} \\
\lim _{\hbar \rightarrow 0}\left\{\hat{E}^{\prime}=e^{-\imath G / \hbar}\left(\imath \hbar \frac{\partial}{\partial t}\right) e^{\imath G / \hbar}\right\}=-\frac{\partial G}{\partial t} \equiv E .
\end{gathered}
$$

Agora, introduzimos a seguinte equação de auto-valor

$$
\left(\frac{\hat{p}^{2}}{2 m}-\hat{E}\right) \psi=\left(\frac{p^{2}}{2 m}-E\right) \psi
$$

Ao usarmos (15) e ao levarmos em conta as relações (1719), obtemos

$$
-\frac{\hbar^{2}}{2 m} \frac{\partial^{2} \psi^{\prime}}{\partial q^{2}}-\frac{\imath \hbar}{2 m} \frac{\partial^{2} \xi}{\partial q^{2}} \psi^{\prime}+\frac{\hbar}{\imath m} \frac{\partial \xi}{\partial q} \frac{\partial \psi^{\prime}}{\partial q}-\imath \hbar \frac{\partial \psi^{\prime}}{\partial t}=0
$$

Ao substituirmos (21) na Eq.(16), chegamos a

$$
\left[\frac{1}{2 m}\left(\frac{\partial G}{\partial q}\right)^{2}+V+\frac{\partial G}{\partial t}\right] \psi=0
$$

Assim, obtemos a equação de Hamilton-Jacobi clássica

$$
\frac{1}{2 m}\left(\frac{\partial S}{\partial q}\right)^{2}+V+\frac{\partial S}{\partial t}=0
$$

em que substituímos $G=G(q, t)$ pela ação clássica $S=$ $S(q, t)$ (ver Eq.(17)). Em princípio, a Eq.(23) é obtida da Eq.(22) para todas as soluções $\psi$ da equação de Schrödinger (3) que satisfazem à nossa equação de auto-valor (20). O importante a ser acentuado é que, para obtermos o limite clássico da equação de Schrödinger, não precisamos calcular explicitamente o limite $\hbar \rightarrow 0$ de certas funções $\psi$, posto que elas não são em geral analíticas nesse limite. Assim, nosso processo de limite clássico funciona bem para funções $\psi$ que podem descrever estados ligados ou não, superpostos ou não, desde que a Eq.(20) seja obedecida.

Diferente da teoria de Bohm e da aproximação WKB, nosso método (15-23) produz corretamente o limite clássico de uma partícula livre, de um oscilador harmônico e de uma partícula em uma caixa para todas as $\psi$, compatíveis com (20), e com momentum $p=\partial S / \partial q \neq 0$. Por razões físicas, a condição $\psi=0$ é desconsiderada porque no domínio clássico os sistemas (oscilador harmônico, partícula livre e em uma caixa) não apresentam regiões nodais onde a probabilidade de achar o sistema é nula.

Em resumo, nosso processo de limite clássico, baseado no limite $\hbar \rightarrow 0$, revela que a representação de Madelung da mecânica quântica não é necessária e adequada para realizar a transição quântico $\rightarrow$ clássico. No Apêndice, aplicamos nosso método à equação de Dirac. A relevância da transformação (15) para calcular o limite clássico da dinâmica quântica é enfatizada também nas Refs.[8,25-29].
Na próxima seção, apresentamos a tradução, diretamente do alemão, de um artigo feito por Einstein, em 1953, sobre a relação entre a física clássica e a física quântica [24]. O leitor interessado pelas discussões entre M. Born, A. Einstein, W. Pauli e D. Bohm sobre as questões conceituais advindas deste trabalho, remetemo-lo às Refs. [17,18,20,21,29$31]$.

\section{IV $O$ artigo de A. Einstein: Considerações elementares sobre a interpretação dos fundamentos da mecânica quântica}

Como característica principal da atual situação da mecânica quântica, considero a seguinte: quanto ao formalismo da teoria, não há nenhuma dúvida, mas quanto à sua interpretação, o mesmo não pode ser dito. Qual a relação que a função $\psi$ possui com as coisas concretas, isto é, com as propriedades individuais de um único sistema? Ou melhor, o que afirma a função $\psi$ com respeito ao "estado real" de um sistema individual?

Antes que continuemos, pode-se duvidar se esta questão possui em geral algum sentido. De fato, pode-se aceitar o seguinte ponto de vista: "real" é apenas aquilo que resulta da própria observação, e não algo existindo objetivamente no espaço e no tempo e independente do ato de observação. Se se aceita este ponto de vista claramente positivista, fica evidente que não é necessário fazer nenhuma consideração de como compreender o "estado real" de um sistema no domínio da teoria quântica. Qualquer esforço parece, pois, ser como uma luta de esgrima contra fantasma.

Esta concepcão nitidamente positivista tem, no entanto, produzido uma fraqueza irreparável: geralmente, ela considera sem sentido todas as proposições verbalmente expressas. Até que ponto é correto tomar como provida de sentido, ou o mesmo, como verdadeira ou falsa, uma descrição de um único resultado de observação? Tal descrição não pode estar baseada em mentiras ou em vivências que experimentamos em sonhos ou em alucinações? A distinção entre estar 
acordado e dormindo possui, em geral, um sentido objetivo? De resto, permanecem como "reais" apenas as experiências emotivas de um Eu sem qualquer possibilidade de asseverar algo sobre elas. Segundo uma análise puramente positivista, os conceitos empregados nas assertivas mostram-se, sem exceção, como desprovidos de sentido.

$\mathrm{Na}$ verdade, os conceitos e os sistemas conceituais independentemente empregados nas nossas proposições são criações humanas, instrumentos auto-criadores, cuja legitimação e valor se baseiam exclusivamente no fato de poderem ordenar "com proveito" as experiências vivenciais (comprovação= Bewährung). Dito de outra forma, estes instrumentos são legítimos na medida em que eles são capazes de "explicar" as experiências de vida ${ }^{1}$.

A legitimidade dos conceitos e dos sistemas conceituais tem de ser avaliada apenas a partir do ponto de vista da sua comprovação. Isto também vale para os conceitos de "realidade física", ou seja, para o conceito de "estado real de um sistema". A priori não existe nenhuma legitimação para postulá-los ou proibí-los como necessários ao pensamento. O que decide é apenas a comprovação. Por trás destes símbolos vocabulares, no desenvolvimento do pensamento físico até o surgimento da teoria quântica, existe um programa que tem se tornado normativo: tudo deve ser reduzido a objetos conceituais, existindo no espaço-tempo, e a relações baseadas em leis que devem ser válidas para estes objetos. Nesta descrição, nada aparece relacionado com um conhecimento empírico referente a estes objetos. Atribui-se à Lua uma posição (relativa a um sistema de coordenadas utilizado) em um determinado instante, independente de as percepções desta posição existirem ou não. Compreende-se este tipo de descrição quando se fala da descrição física de um "mundo externo real” ou de seus constituintes básicos (pontos materiais, campo etc.).

A legitimação deste programa não foi seriamente posta em dúvida pelos físicos, pois pareceu que tudo que surgia em tal descrição, em princípio, poder-se-ia verificar empiricamente para algum caso particular. Que isto não passa de uma ilusão, foi primeiro demonstrado por Heisenberg de um modo convincente para os físicos no tocante aos fenômenos quânticos.

Desde então, o conceito de "realidade física" tornou-se problemático. Surgiu, assim, a questão: no fundo, o que a física teórica (por meio da mecânica quântica) procura descrever? e qual sua relação com as leis físicas já estabelecidas? Esta questão foi respondida de modos bem diversos.

A fim de chegar a uma resposta, levemos em conta o que a mecânica quântica tem a dizer sobre sistemas macroscópicos, i.é, sobre objetos que temos a sensação de serem "diretamente perceptíveis". De fato, sabemos que tais objetos e as leis que os governam são representados pela física clássica com uma certeza quase apodídica. Não duvidamos que exista para tais objetos em cada instante uma configuração espacial (posição) como também uma velocidade (ou impulso), isto é, uma situação real. Isto decorre, como aproximação, da estrutura da mecânica quântica.

Perguntamos: a mecânica quântica implica, por aproximação, a descrição real fornecida pela mecânica clássica para corpos macroscópicos? Ou, caso esta pergunta não possa ser respondida com um simples "sim", em que sentido ela o faz? Queremos abordar este problema com base em um exemplo concreto.

\section{IV.1 O exemplo}

O sistema consiste de uma esfera com aproximadamente $1 \mathrm{~mm}$ de diâmetro que se move entre duas paredes paralelas (distantes mais ou menos $1 \mathrm{~m}$ uma da outra) ao longo do eixo $x$ de um sistema de coordenadas. Os choques são idealmente elásticos. Neste sistema macroscópico idealizado, imaginamos que as paredes possam ser substituídas por uma energia potencial bem definida em termos apenas das coordenadas dos pontos materiais que constituem a esfera. Supõe-se também que os processos de reflexão não produzam nenhum acoplamento entre a coordenada $x$ do centro de gravidade da esfera e suas coordenadas "internas" (inclusive as coordenadas de ângulo). Assim, para os nossos propósitos a posição da esfera (abstraindo-se de seu raio) pode ser descrita apenas por $x$.

Segundo a mecânica quântica, trata-se de um processo com energia fixa. A onda de de Broglie (a funcão $\psi$ ) é, pois, harmônica no tempo. $\psi$ é diferente de zero apenas entre $x=-\ell / 2$ e $x=+\ell / 2$. Nos pontos extremos do trajeto, o caráter contínuo é obtido ao se exigir que a função $\psi$ seja nula fora da região permitida para o movimento da esfera e $\operatorname{nos}$ pontos $x= \pm \ell / 2$.

A função $\psi$ é, portanto, uma onda estacionária que pode ser representada, entre $x=-\ell / 2$ e $x=+\ell / 2$, pela superposição de duas ondas harmônicas que se propagam em direções opostas:

$$
\psi=\frac{1}{2} A e^{\imath(a t-b x)}+\frac{1}{2} A e^{\imath(a t+b x)}
$$

ou

$$
\psi=A e^{\imath a t} \cos (b x) .
$$

Da Eq.(25), vê-se que o fator $A$ tem de ser escolhido como sendo igual em ambos os membros de (24) para que as condições de contorno possam ser satisfeitas nos pontos $x= \pm \ell / 2$. Sem perda de generalidade, $A$ pode ser considerado real. Segundo a equação de Schrödinger, $b$ é determinado completamente pela massa $m$. Ao fator $A$ nada é acrescido ao que já é conhecido.

Para que seja frutífera a comparação do exemplo acima com o correspondente problema clássico, temos ainda de estabelecer que o comprimento de onda de de Broglie $2 \pi / b$ seja pequeno em comparação com $\ell$.

Começamos com o significado da função $\psi$ baseandonos na interpretação usual, dada por Born, em termos de probabilidade:

$$
W=\int \psi \bar{\psi} d x=A^{2} \int \cos ^{2}(b x) d x .
$$

Esta é a probabilidade para que a coordenada $x$ do centro de gravidade da esfera esteja localizada em um dado intervalo $\triangle x$. Ela é - abstraindo-se de uma "estrutura fina" de caráter ondulatório [nodos, nos quais $W=0$ ] de cuja realidade física não se duvida - simplesmente cte. $\triangle x$.

\footnotetext{
${ }^{1}$ A semelhança linguística dos conceitos "verdadeiro" ("wahr") e "dar bom resultado" ("sich bewähren") constitui um parentesco fundamental, apenas com a ressalva de que isto não pode ser compreendido de um modo utilitarista.
} 
E quanto à probabilidade dos valores do impulso (ou velocidade) da esfera? Estas probabilidades são obtidas pela expansão de Fourier de $\psi$. Se (24) fosse válida de $-\infty$ até $+\infty$, então, (24) já seria a expansão de Fourier desejada. Haveria, portanto, dois valores para o impulso igualmente bem determinados em direções opostas e com a mesma probabilidade. Mas, pelo fato de as duas ondas serem confinadas, elas fornecem para cada membro uma transformada de Fourier contínua com domínio espectral tão estreito quanto maior o número de comprimentos de onda de Broglie contidos na região $\ell$. Isto segue do fato de serem possíveis apenas dois valores bem definidos do impulso em direções opostas. Estes valores coincidem com os do caso clássico, ambos com igual probabilidade.

Portanto, estes dois resultados estatísticos são, afora os pequenos desvios ocasionados pela estrutura quântica, os mesmos válidos para um "ensemble no tempo" de sistemas no caso da teoria clássica. Isto acontece desde que a teoria seja inteiramente satisfatória.

Mas, agora levantemos a pergunta: pode esta teoria fornecer uma descrição realista de um caso individual? Esta pergunta terá de ser respondida com um "não". Para fundamentar esta decisão, é essencial que o sistema individual seja um sistema "macroscópico", pois, neste caso estamos convictos de que ele esteja em todo instante em um "estado real" que é aproximada e corretamente descrito pela mecânica clássica. O sistema macroscópico, do tipo considerado por nós, tem assim em todo instante uma determinada posição (a coordenada de seu centro de gravidade) - ou pelo menos o valor médio sobre um curto intervalo de tempo - e um determinado impulso (a menos de um sinal). Nenhuma destas duas características resulta da funcão $\psi$ (24). Com a ajuda da interpretação de Born, a partir de $\psi$ deduzem-se apenas características que se relacionam com um ensemble estatístico de sistemas do tipo em consideração.

O exemplo, acima estudado, mostra que nem toda função $\psi$, solução da equação de Schrödinger, corresponde de modo aproximado à uma descrição realista no sentido da mecânica clássica. Isto fica explícito quando se leva em conta uma função $\psi$ que se origina da superposição de duas soluções do tipo (24), cujas freqüências (ou energias) são bem diferentes uma da outra. A uma tal superposição, portanto, não corresponde nenhum sistema real da mecânica clássica (mas sim, um ensemble estatístico de tais sistemas reais no sentido da interpretação de Born).

À guisa de generalização, concluímos: a mecânica quântica descreve ensembles de sistemas, e não um sistema individual. A descrição por meio da função $\psi$ constitui, neste sentido, uma descrição incompleta de um único sistema; não é portanto uma descrição realista do estado do sistema.

Observação: poder-se-ia levantar a seguinte objeção à nossa conclusão. O caso considerado por nós, em que a função $\psi$ possui uma freqüência extremamente bem definida, é um caso extremo para o qual, talvez, a semelhança exigida com o problema clássico pudesse ser desconsiderada de modo excepcional. Se se admite um domínio finito e também pequeno para a frequiência temporal, então pode-se obter, por meio da escolha aproximada das amplitudes e das fases das funções $\psi$ superpostas, que a função $\psi$ resultante possui aproximadamente posição e impulso bem definidos. Segundo este ponto de vista, não se poderia pro- curar restringir àquelas funções $\psi$ que são apropriadas e assim obter funções $\psi$ que pudessem ser compreendidas como representação de um único sistema?

Uma tal possibilidade tem de ser posta de lado com base no fato de não se manter com o passar do tempo uma tal situação com posição bem definida.

Por causa de a equação de Schrödinger e de sua interpretação, dada por Born, não levarem a uma descrição realista dos estados de um único sistema, estimula-se naturalmente a procura por uma teoria desprovida de tal limitação.

No momento, há duas tendências nesta direção; ambas se fundamentam na equação de Schrödinger, no entanto, uma leva em consideração a interpretação de Born e a outra não. A primeira remonta à idéia de de Broglie, posteriormente retomada com muita perspicácia por Bohm.

Tal como na investigacão original de Schrödinger, onde a equação de onda é deduzida usando analogia com a mecânica clássica (a linearizacão da equação de Jacobi da mecânica clássica), assim deve também estar fundamentada, por analogia, a equação de movimento de um sistema individual quantizado, descrito por uma solução da equação de Schrödinger. $\mathrm{O}$ procedimento é o seguinte: coloca-se $\psi$ na forma

$$
\psi=R e^{\imath S}
$$

da qual resulta que $R$ e $S$ são funções reais em termos das coordenadas. A derivada de $S$ com respeito à posição, que dá o impulso ou a velocidade do sistema, deve implicar a evolução temporal do sistema individual.

Uma olhada na Eq.(25) mostra que em nosso caso $\partial S / \partial x$, e também a velocidade, se anulam. De acordo com o nosso exemplo, este resultado é muito importante para levantar uma objeção, já apontada por Pauli há cerca de 25 anos, a esta teoria [de de Broglie-Bohm]. O fato de ser nula a velocidade vai de encontro com a exigência bem estabelecida de que no caso de sistemas macroscópicos o movimento deve coincidir aproximadamente com o movimento descrito pela mecânica clássica.

A segunda tentativa de fundamentar a equação de Schrödinger, como uma descrição realista de um único sistema, remonta aos primeiros estudos realizados por Schrödinger, cuja idéia é resumidamente a seguinte: a função $\psi$ representa a realidade e não necessita da interpretação de Born. O quadro (Gebilde) pictórico que se faz dos átomos, sobre o qual o campo $\psi$ devia dizer algo, simplesmente não existe, pelo menos não como um quadro localizado. No caso do nosso sistema macroscópico, isto significa: este sistema, de fato, não existe como tal; em todas as situações, não existe nem de modo aproximado algo como sua posição em determinado tempo. Aqui também é violada, portanto, a condição de que a descrição quântica do movimento de um sistema macroscópico tem de coincidir por aproximacão (angenähert) à correspondente descrição fornecida pela mecânica clássica.

O que resulta da nossa investigação é o seguinte: a única interpretação até agora aceitável da equação de Schrödinger é aquela dada pela interpretação estatística de Born. Contudo, esta interpretação não fornece nenhuma descrição realista de um único sistema; ao contrário, ela apenas versa sobre asserções estatísticas de ensembles de sistemas.

Na minha opinião, por princípio, não é satisfatório sustentar uma tal concepção da Física, visto que a descrição 
objetiva dos sistemas macroscópicos individuais ("descrição realista do estado") não pode ser desprezada sem que a compreensão física do mundo se dissolva de certa forma em um nevoeiro. Finalmente, a concepção inevitável é que a Física tem de fomentar uma descrição realista de um único sistema. A natureza como um todo pode ser pensada apenas como sistema individual (existindo uma vez só, sem necessidade de repetição) e não como um "ensemble de sistemas".

\section{Agradecimentos}

O autor agradece ao Prof. Waldyr A. Rodrigues Jr. (IMECC-UNICAMP) pelo apoio científico e à Fundação de Amparo à Pesquisa do Estado de São Paulo (FAPESP, contrato no. 99/11593-3) pelo apoio financeiro.

\section{Apêndice: Limite clássico da equação de Dirac}

Uma partícula com spin $1 / 2$, massa $m$ e carga $e$ na presença de campos eletromagnéticos $A_{\mu}=A_{\mu}\left(q^{\nu}\right)$ (métrica (+,-,-,-)) é descrito pela equação de Dirac [11]

$$
\gamma^{\mu}\left[\hbar \frac{\partial}{\partial q^{\mu}}-\frac{\imath e}{c} A_{\mu}\right] \psi+m c \psi=0 .
$$

( $c$ é a velocidade da luz, $\gamma^{\mu}$ as matrizes de Dirac $4 \times 4$ e $\psi$ uma matriz spinorial $4 \times 1$ ). Ao efetuarmos sobre (26) a transformação unitária

$$
\psi^{\prime}=e^{-\imath \xi / \hbar} \psi
$$

obtemos

$$
\gamma^{\mu}\left[\hbar \frac{\partial}{\partial q^{\mu}}+\imath \frac{\partial \xi}{\partial q^{\mu}}-\frac{\imath e}{c} A_{\mu}\right] \psi^{\prime}+m c \psi^{\prime}=0
$$

Ao usarmos o resultado

$$
\lim _{\hbar \rightarrow 0}\left[e^{-\imath \xi / \hbar}\left(\frac{\hbar}{\imath} \frac{\partial}{\partial q^{\mu}}\right) e^{\imath \xi / \hbar}\right]=\frac{\partial \xi}{\partial q_{\mu}}=p^{\mu},
$$

a transformação (27) sobre a equação de autovalor

$$
\frac{\hbar}{\imath} \frac{\partial \psi}{\partial q_{\mu}}-p^{\mu} \psi=0
$$

leva a

$$
\frac{\partial \psi^{\prime}}{\partial q_{\mu}}=0
$$

Inserimos (31) em (28) e, em seguida, quadramos. Chegamos, assim, à equação de Hamilton-Jacobi relativística clássica

$$
\left[\frac{\partial S}{\partial q^{\mu}}-\frac{e}{c} A_{\mu}\right]^{2}+m^{2} c^{2}=0 .
$$

A propósito, gostaríamos de ressaltar que a teoria de Bohm pode ser inadequada para calcular o limite clássico da equação de Dirac [32].

\section{Referências}

[1] S. Borowitz, Fundamentals of Quantum Mechanics (W. A. Benjamin, New York, 1967); S. M. Blinder, Foundations of Quantum Mechanics (Academic Press, London, 1974); R. Eisberg and R. Resnick, Quantum Physics (John Wiley and Sons, New York, 1974); P. Fong, Elementary Quantum Mechanics (Addison-Wesley, Reading, 1962); S. Gasiorowicz, Quantum Physics (John Wiley and Sons, New York, 1974); K. Gottfried, Quantum Mechanics (W. A. Benjamin, New York, 1966); J. L. Powell and B. Crasemann, Quantum Mechanics (Addison-Wesley, Reading, 1961); E. C. Kemble, The Fundamental Principles of Quantum Mechanics (Dover, New York, 1937); D. I. Blokhintsev, Mécanique Quantique (Masson, Paris, 1967); R. Shankar, Principles of Quantum Mechanics (Plenum Press, New York, 1980); L. Schiff, Quantum Mechanics (McGraw-Hill, Tokyo, 1955); A. Messiah, Mécanique Quantique (Dunod, Paris, 1958); E. Merzbacher, Quantum Mechanics (Wiley, New York, 1961); C. Cohen-Tannoudji, F. Diu, F. Laloë, Mécanique Quantique (Hermann, Paris, 1977); J. J. Sakurai, Modern Quantum Mechanics (Addison-Wesley, New York, 1994).

[2] P. Ehrenfest, "Bemerkung über die angenäherte Gültigkeit der klassischen Mechanik innerhalb der Quantenmechanik", Z. Phys. 45, 455-457 (1927). [Traduzimos este artigo na Ref.[9]].

[3] G. Wenzel, "Eine Verallgemeinerung der Quantenbedingungen für die Zwecke der Wellenmechanik", Z. Phy. 38, 518529 (1926).

[4] H. Kramers, "Wellenmechanik und halbzahlige Quantisierung”, Z. Phys. 39, 828-840 (1926).

[5] L. Brillouin, "Remarques sur la mécanique ondulatoire", Journal de Physique et Radium 6, 333-363 (1926).

[6] L. E. Ballentine, Y. Yang, and J. P. Zibin, "Inadequacy of Ehrenfest's theorem to characterize the classical regime", Phys. Rev. A 50, 2854-2859 (1994).

[7] L. E. Ballentine, Quantum Mechanics (World Scientific, New York, 1998).

[8] A. O. Bolivar, "Classical limit of bosons in phase space", Physica A 315, 601-615 (2002).

[9] A. O. Bolivar, "Teorema de Ehrenfest e o limite clássico da mecânica quântica", Rev. Bras. Ens. Fís. 23 (2), 190-195 (2001).

[10] A. A. Grib and W. A. Rodrigues, Jr., Nonlocality in Quantum Physics (Kluwer, New York, 1999).

[11] W. Pauli, General Principles of Quantum Mechanics (Springer-Verlag, Berlin, 1980).

[12] D. Bohm, Quantum Theory (Prentice-Hall, New York, 1951).

[13] J. H. van Vleck, "The correspondence principle in the statistical interpretation of quantum mechanics", Proc. N. A. S. 14, 178-188 (1928).

[14] L. S. Brown, "Classical limit and the WKB approximation", Am. J. Phys. 40, 371-376 (1972); D. Home and S. Sengupta, "Classical limit of quantum mechanics", Am. J. Phys. 51, 265-267 (1983).

[15] E. Madelung, "Quantentheorie in hydrodynamischer Form", Z. Phys. 40, 322-326 (1927). 
[16] D. Bohm, "A suggested interpretation of the quantum theory in terms of 'hidden' variables, I and II", Phys. Rev. 85, 166193 (1952).

[17] D. Bohm and B. J. Hiley, "Unbroken quantum realism, from microscopic to macroscopic levels", Phys. Rev. Lett. 55, 2511-2514 (1985).

[18] D. Bohm, B. J. Hiley, and P. N. Kaloyerou, "An ontological basis for the quantum theory", Phys. Rep. 144, 321-375 (1987).

[19] P. R. Holland and K. A. Kyprianidis, "Quantum potential, uncertainty and the classical limit", Ann. Inst. Henri Poincaré 49, 325-339 (1988).

[20] D. Bohm and B. J. Hiley, The Undivided Universe, (Routledge, London and New York, 1993).

[21] P. R. Holland, The Quantum Theory of Motion (Cambridge University Press, Cambridge, 1993).

[22] J. Cohn, "Quantum theory in the classical limit", Am. J. Phys. 40, 463-467 (1972).

[23] N. Rosen, "The relation between classical and quantum mechanics", Am. J. Phys. 32, 597-600 (1964); id., "Quantum particles and classical particles", Found. Phys. 16, 687-700 (1986).

[24] A. Einstein, "Elementare Überlegungen zur Interpretation der Grundlagen der Quanten-Mechanik", in Scientific Papers Presented to Max Born on his Retirement from the Tait Chair of Natural Philosophy in the University of Edinburgh, pp.3340, (Oliver and Boyd, Edinburgh, 1953).

[25] A. O. Bolivar, "Quantization and classical limit of a linearly damped particle, a van der Pol system and a Duffing system”, Random Operators and Stochastic Equations 9 (3), 275-286 (2001).

[26] A. O. Bolivar, "Classical limit of fermions in phase space", J. Math. Phys. 42, 4020-4030 (2001).

[27] A. O. Bolivar, "The Wigner representation of classical mechanics, quantization and classical limit", Physica A 42, 219240 (2001).

[28] A. O. Bolivar, "Dynamical quantization and classical limit", Can. J. Phys. (2003) (a ser publicado).

[29] A. O. Bolivar, The Quantum-Classical Correspondence: Dynamical Quantization and Classical Limit (Springer Verlag, Berlin, Heidelberg, 2003), em preparação.

[30] A. Einstein, in The Born-Einstein Letters (Macmillan, London, 1971).

[31] M. Born, "Continuity, determinism and reality", Det Kongelige Danske Videnskabernes Selskab Matematisk-fysiske Meddlelelser 30, 1-26 (1955).

[32] J. E. Maiorino and W. A. Rodrigues Jr., What is Superluminal Wave Function?, a ser publicado. 